\title{
Models of Radar Imaging of the Ocean Surface Waves
}

\author{
CHARLES ELACHI, MEMBER, IEEE, AND WALTER E. BROWN, JR.
}

\begin{abstract}
A number of models which would explain ocean wave imagery taken with a synthetic aperture imaging radar are analyzed analytically and numerically. Actual radar imagery is used to support some conclusions. The models considered correspond to three sources of radar backscatter cross section modulation: tilt modulation, roughness variation, and the wave orbital velocity. The effect of the temporal changes of the surface structure, parametric interactions, and the resulting distortions are discussed.
\end{abstract}

\section{INTRRODUCTION}

$\mathrm{E}^{\mathrm{x}}$ XPERIMENTAL observations undertaken with the Jet Propulsion Laboratory's $L$-band and VHF airborne imaging radars [1]-[4] and the Naval Research Laboratory's L-band imaging radar [5], [6] have demonstrated that ocean surface waves and patterns can be imaged using a synthetic aperture radar. Radar imagery of a wide range of ocean surface patterns have been obtained and in some cases confirmed by optical photography or ground truth [3], [4]. Specifically ocean swells, oil slicks, wind slicks, currents, internal waves, eddies, and ship wakes have been imaged under a variety of conditions.

The physics of radar wave scattering from the ocean surface is generally known as related to radar scatterometry. However in radar imaging, two new major factors have to be accounted for: 1) the high resolution capability of the imaging radar (a few meters to a few tens of meters), and 2) the use of coherent Doppler information to generate the image. The impact and importance of these two factors on the formation of ocean surface imagery is still not well understood, and a number of concepts have been proposed to explain how a radar image of the ocean surface is generated.

The radar image is a two-dimensional presentation of the variation of the local coherent backscatter cross section of the surface. In the case of the ocean, three sources of cross section modulation seem to play an important role: change of the local tilt angle, variation of the surface roughness, and the waves' orbital velocity. The last type of modulation affects the backscatter cross section indirectly because of the way an imaging radar generates a high resolution image. In this paper we will discuss in detail these three types of modulation and evaluate their effect using analytical and numerical models. We also present experimental data that supports or in some cases contradicts one or more of the above theories.

Manuscript received September 11, 1975; revised May 27, 1976. This paper represents the results of one phase of research carried out at JPL, California Institute of Technology, under contract NAS7-100, sponsored by the National Aeronautics and Space Administration.

The authors are with the Space Science Division, Jet Propulsion Laboratory, California Institute of Technology, Pasadena, CA 91103.
The field of radar imagery of the ocean is a new field of research and much work is still needed to fully understand the content of the radar image. The objective of this paper is to make a first step in this direction, and to review different ideas proposed by scientists interested in this field, not to present final proven theories.

The principle of imaging radar will be discussed in this paper only as related to the theories considered. A detailed review of imaging radars can be found in many books [7]-[9]. In Fig. 1 we show the geometry for the imagery that will be presented in this paper. This imagery was taken with the JPL $25-\mathrm{cm}$ imaging radar in the $\mathrm{HH}$ polarization mode, i.e., the transmitted and received electric vectors are perpendicular to the plane of incidence.

\section{Theories and Principle of Radar Imaging of OCEAN WAVES}

Radar wave scattering from the ocean surface has been studied both theoretically and experimentally for many years $[10]-[15]$. The scattering properties can be explained using a composite model where specular scattering (i.e., geometric optics) is dominant near nadir at incidence angles less than $15^{\circ}$, and Bragg type scattering is dominant at larger incidence angles. The Bragg scattering is basically a resonant reflection from surface waves of wave number $K_{1}=2 k \sin \theta$, where $k=2 \pi / \lambda$ is the radar wave number, $\lambda$ is the radar wavelength and $\theta$ is the incidence angle. The most recent Bragg scattering theories also account for the local tilt of the surface [16]-[19]. Thus the backscatter cross section is an integral that includes the contribution of all possible slopes from water waves and swells that are present during observation. In the case of a fully developed sea with no swells, the integration is usually carried over the wave numbers $K_{2}<K<K_{1}$, where $K_{2}=g U^{-2}, U$ is the mean wind velocity, and $g$ is the gravity acceleration. This integration is valid because the resolution element in usual scattering experiments is large relative to most of the ocean waves.

However, this is not the case in imaging radar experiments where typical resolutions are about 10 to $30 \mathrm{~m}$, which is less than the wavelength of many wind driven waves and basically all swells. Thus in deriving the properties of the radar image, the ocean spectrum can be divided into three regions (Fig. 2).

1) $K=K_{1}=2 k \sin \theta$. This is the resonant region that generates the backscattered wave. For most imaging radars presently available $\lambda \leq 2 \mathrm{~m}$, thus $K \gtrsim 6 \sin \theta \mathrm{m}^{-1}$, i.e., $\Lambda=2 \pi / K \lesssim 4 / \sin \theta \mathrm{m}$. This is basically the short gravity waves region. It should be mentioned that for high fre- 


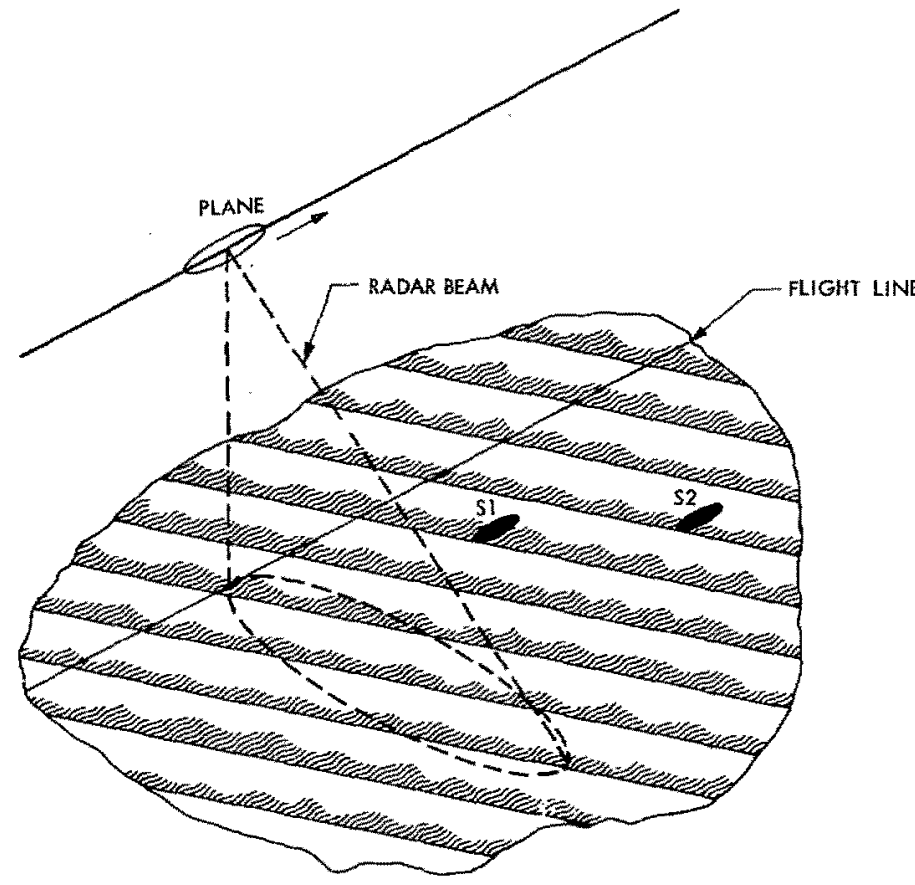

(a)

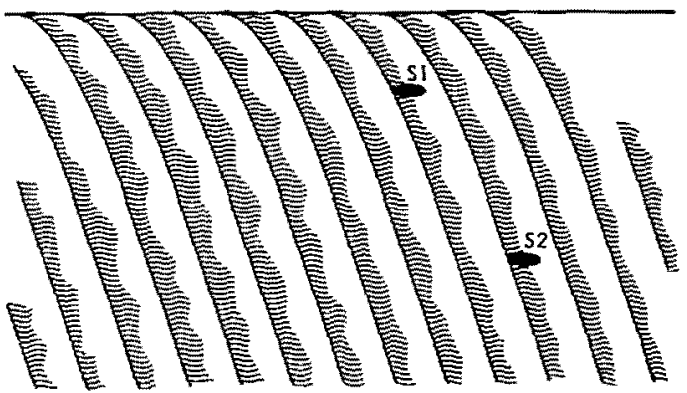

(b)

Fig. 1. Geometry of radar imagery. (a) Actual flight geometry, (b) Geometry of radar inage. Bright line in image corresponds to nadir specular return, and therefore it corresponds to line of flight. As radar measures time delay in range, image is usually distorted, i.e., linear features are bent near nadir. However, this distortion can be easily corrected by standard digital techniques.

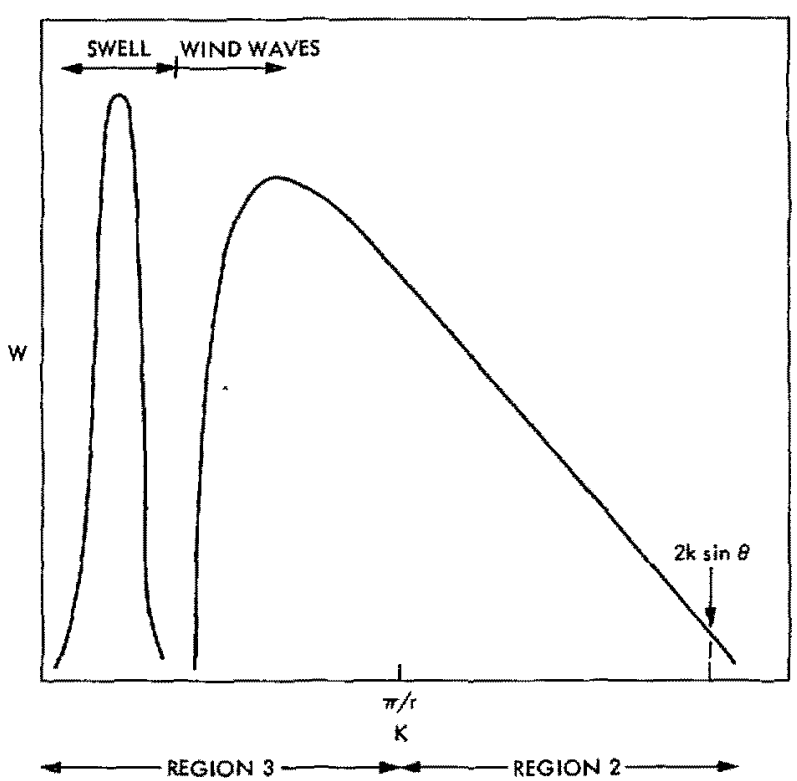

Fig. 2, Sketch of ocean wave spectrum showing three regions of importance for imaging radar. Bragg scattering results from waves where $K=2 k \sin \theta$. Waves in region 2 are smaller than radar resolution, thus each image pixel is average over these waves: Waves in region 3 are large enough to be imaged individually, $r$ is radar resolution.

quency radars ( $\lambda$ of the order of $1 \mathrm{~cm}$ ) the Bragg scattering results mainly from the capillary waves.

2) $2 \pi / 2 r<K<\infty$, where $r$ is the radar image resolution. The waves in this region cannot be individually seen, thus each resolution element in the radar image corresponds to the composite backscatter cross section, which accounts for

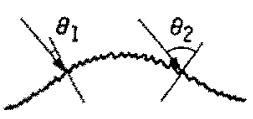

(a)

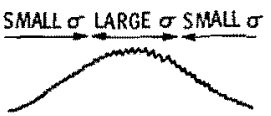

(b)

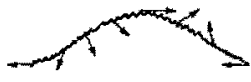

(c)

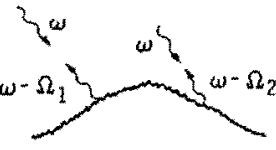

(d)
Fig. 3. Different models for formation of radar image of ocean waves. Basic backscatter is assumed to be of Bragg type. (a) Tilt modulation which leads to modulation of actual incidence angle and backscatter cross section. (b) Roughness modulation which leads to modulation of backscatter cross section. (c) Orbital velocity which leads to periodic Doppler shift and, in turn, periodic displacement, and defocusing of surface units. (d) Parametric effects which lead to periodic frequency shift and defocusing. In actuality, all these effects might be contributing at the same time to image formation. Scales in figures are exaggerated for illustration.

the contribution of all possible slopes of the water waves in this region of the spectrum. For a radar image resolution of $25 \mathrm{~m}$, this spectral region covers all waves of wavelength up to $\Lambda \simeq 50 \mathrm{~m}$. The factor 2 is included because at least two properly located pixels are needed to discriminate a change in the image brightness.

3) $K<2 \pi / 2 r$. All waves in this region have a wavelength larger than two resolution elements, therefore each ocean wave could be individually imaged as such. This covers all swells and wind waves where the local wind is relatively large (i.e., about $18 \mathrm{knots}$ or more for $r=25 \mathrm{~m}$ ).

The radar image is a two-dimensional representation of the surface backscatter cross section. Thus a long wave 


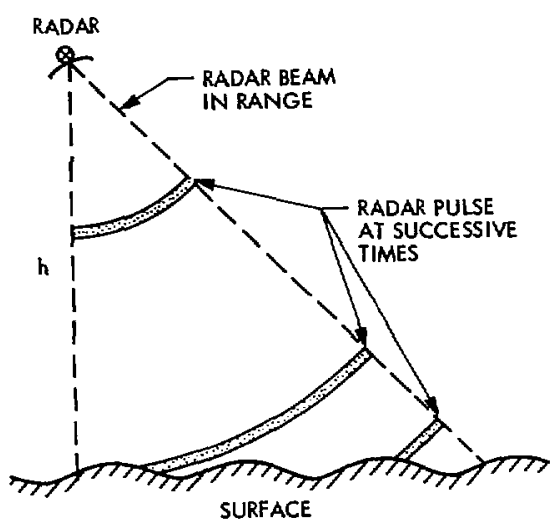

(a)
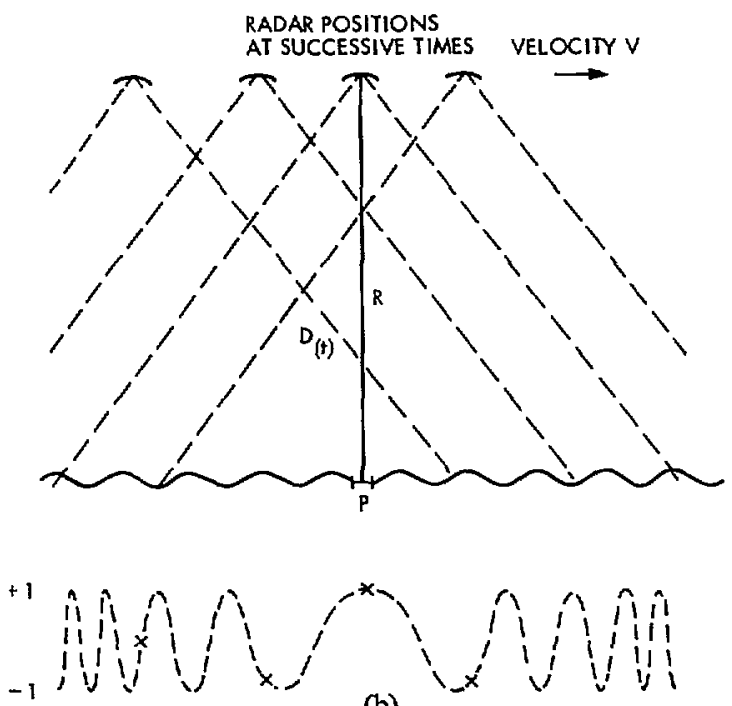

(b)

Fig. 4. Principle of synthetic aperture imaging radar. (a) Range geometry. Plane of figure is perpendicular to flight line. Scattered echo at successive time corresponds to different sections of surface or range bins. (b) Azimuth plane or along flight line. Signal history (i.e., real part of signal) from point $P$ changes as shown in sketch in bottom. Phase $\Phi(t)=4 \pi D(t) / \lambda$. This signal curve characterizes point $P$. Neighboring resolution element will have similar but displaced signal history. Thus simultaneous processing of range information (time delay) and azimuth information (phase history) would allow positioning of $P$ in two dimensions. Strength of corresponding echo would then give brightness of image at that point.

could be imaged if it is capable of modulating the backscatter cross section. This leads us to the first two theories that might explain the radar imagery: 1) the surface slope modulation of a homogeneously rough surface, and 2) the surface roughness modulation. Each theory is based on one of the two surface parameters that affect the Bragg scattering: the local incidence angle, and the magnitude of the surface perturbation. (See Fig. 3.)

If we consider a number of resolution elements across one period of a long wave, it is easy to see that usually the normal to the surface at each resolution element forms a different angle relative to any line of sight, i.e., a different local incidence angle (Fig. 3(a)). The modulation of the incidence angle is maximum when the waves propagate perpendicular to the flight line of the radar platform, and is minimum when the wave propagates parallel to it. Thus for this theory, the capability of the radar to image ocean waves is very much dependent on the angle between the wave propagation vector and the flight line. (The resulting modulation of the backscatter cross section is derived in Section III.)

It has been demonstrated theoretically and experimentally that the small scale roughness is not necessarily homogeneous across a water wave [20]-[24]. Surface straining, local wind, wave breaking, nonlinear coupling, and other effects could lead to a variation of the population of the short gravity waves across a long wave period, which leads to a modulation of the backscatter cross section (Fig. 3(b)). This model is discussed in Section IV.

The third theory which seems also to have some popularity is based on the effect of the orbital velocity of long waves. Before we further discuss this concept, we must explain how the high resolution radar image is generated. In Fig. 4 we show in a simple way the principle of an imaging radar. A short pulse is transmitted toward the surface. The spread out echo is received. Each part of the echo corresponds to a different range bin. Thus the surface can be divided in range bins where each corresponds to a different time delay. Very high resolution can be obtained by having a very short pulse. In the azimuth direction, high resolution is a result of the coherency of the system. Each received echo is mixed with the coherent onboard local oscillator such that the phase history of the echo can be deduced. To illustrate, let us consider a resolution element $P$ on the surface. As the platform flies by it, the real part of the echo follows a specific pattern (Fig. 4) that characterizes that specific resolution element. The echo is recorded (usually on film and sometimes on tape) and later compressed to generate a point or a pixel in the image that corresponds to that specific resolution element. The echo phase history is a function of the velocity of the platform, and is equal to (Fig. 4)

$\Phi(t)=\frac{4 \pi}{\lambda} D(t)=\frac{4 \pi}{\lambda}\left(R^{2}+v^{2} t^{2}\right)^{1 / 2} \simeq \frac{4 \pi R}{\lambda}+\frac{2 \pi v^{2} t^{2}}{\lambda R}$

where $R$ is the normal distance from the flight line to the resolution element, $v$ is the platform velocity, and $t$ is the time. Usually the radar is pulsed, thus $\Phi$ gives the locus of the phase of the echo from pulse to pulse.

If the resolution element is moving in the direction of the platform motion with a velocity $V_{p} \ll v$, then (1) becomes

$$
\Phi(t) \simeq \frac{4 \pi R}{\lambda}+\frac{2 \pi v^{2} t^{2}}{\lambda R} \pm \frac{4 \pi}{\lambda R} v V_{p} t^{2}
$$

Thus if there is a modulation if the velocity $V, \Phi(t)$ will be modulated, leading to a bunching effect that would generate the image even if the surface has a uniform scatterers distribution. This model will be discussed in more detail in Section VIII. The effect of the temporal change of the surface structure and its motion are left to Section VI, and parametric interaction effects are discussed in Section VII. The different models discussed are sketched in Fig. 3. 


\section{THLt Modulation Model}

Let us consider a slightly rough patch of the ocean that is characterized by its energy spectrum $W\left(K_{x}, K_{y}\right)$ and the local tilt angles $\psi$ and $\delta$, where $\psi$ is the tilt angle in, and $\delta$ is the tilt angle perpendicular to the plane of incidence. The first-order normalized radar backscatter cross section of this patch is [17], [19]

$\sigma\left(\theta_{i}\right)=4 \pi k^{4}|\alpha| W(2 k \sin (\theta-\psi), 2 k \cos (\theta-\psi) \sin \delta)$

where $\theta_{i}$ is the local incidence angle, $\theta$ is the angle at the radar platform between the vertical and the line of observation, and

$$
\begin{gathered}
\alpha=\cot ^{4} \theta_{i}\left\{\begin{array}{c}
{\left[\sin ^{2}(\theta+\psi)\left(\cos ^{2} \delta\right) T_{1}+\left(\sin ^{2} \delta\right) T_{2}\right]^{2},} \\
\text { for HH polarization } \\
{\left[\sin ^{2}(\theta+\psi)\left(\cos ^{2} \delta\right) T_{2}+\left(\sin ^{2} \delta\right) T_{1}\right]^{2},} \\
\text { for VV polarization }
\end{array}\right. \\
T_{1}=\frac{\varepsilon-1}{\left[\cos \theta_{i}+\left(\varepsilon-\sin ^{2} \theta_{i}\right)^{1 / 2}\right]^{2}} \\
T_{2}=\frac{(\varepsilon-1)\left[\varepsilon\left(1+\sin ^{2} \theta_{i}\right)-\sin ^{2} \theta_{i}\right]}{\left[\varepsilon \cos \theta_{i}+\left(\varepsilon-\sin ^{2} \theta_{i}\right)^{1 / 2}\right]^{2}} \\
\cos \theta_{i}=\cos \delta \cos (\theta-\psi)
\end{gathered}
$$

where $\varepsilon$ is the dielectric constant of the surface.

The tilt angles $\delta$ and $\psi$ can be written as

$$
\begin{aligned}
& \delta=\delta_{1}+\delta_{2} \\
& \psi=\psi_{1}+\psi_{2}
\end{aligned}
$$

where $\delta_{1}$ and $\psi_{1}$ are the resulting tilts from all waves of wavelengths less than twice the resolution of the radar, i.e., waves that cannot be discriminated, and $\delta_{2}, \psi_{2}$ are the resulting tilts from the larger waves. Thus in computing the backscatter from a resolution element, i.e., brightness of one image pixel, we have to integrate over all possible values of $\delta_{1}$ and $\psi_{1}$. This gives

$$
\sigma\left(\theta, \psi_{2}, \delta_{2}\right)=\int_{-\infty}^{+\infty} \int_{-\infty} \sigma\left(\theta_{i}\right) p\left(\tan \delta_{1}, \tan \psi_{1}\right) d\left(\tan \delta_{1}\right) d\left(\tan \psi_{1}\right)
$$

where $p\left(\tan \delta_{1}, \tan \psi_{1}\right)$ is the probability density of slopes for water waves that are smaller than twice the radar resolution. Cox and Munk [25] found the slopes in the sea to be approximately Gaussian, thus [26]

$$
\begin{gathered}
p\left(\tan \delta_{1}, \tan \psi_{1}\right)=\frac{1}{2 \pi \Delta^{1 / 2}} \exp \\
\left\{-\frac{m_{02} \tan ^{2} \psi_{1}-2 m_{11} \tan \delta_{1} \tan \psi_{1}+m_{20} \tan ^{2} \delta_{1}}{2 \Delta}\right\} \\
\Delta=m_{02} m_{20}-m_{11}^{2}
\end{gathered}
$$

where $m_{p q}$ is the $(p, q)$ th moment of the surface elevation energy spectrum.

Many spectra were proposed to describe the ocean surface. However, for the purpose of illustration and numerical calculation we used a Pierson-Moskowitz wavevector spectrum that is isotropic for short waves (centimeter scale), and a $\cos ^{2} \gamma / 2$ directional spectrum for medium size waves (meter scale). Thus in evaluating (7) we assume

$$
W(K, y)=\frac{\beta}{2 \pi K^{4}} \cos ^{2}\left(\frac{\gamma}{2}\right) e^{-v\left(K_{c} / K\right)^{2}}
$$

where $\beta \simeq 8 \times 10^{-3}, v \simeq 0.74, K_{c}=g / U^{2}$, and $\gamma$ is the direction. However, for the expression of $W$ in (3), we took

$$
W\left(K_{x}, K_{y}\right)=\beta / 2 K^{4}
$$

where $K^{2}=K_{x}{ }^{2}+K_{y}{ }^{2}$. The reason we dropped the exponential is that for practically all imaging radars the Bragg resonance wavevector $K_{1}$ is $\gg K_{c}$ except in very light wind. For instance for $\lambda=25 \mathrm{~cm}$ and $\theta=30^{\circ}$, then $K_{1}=25 \mathrm{~m}^{-1}$. An equal $K_{c}$ would correspond to approximately a 1.2 knot wind.

From (8) we can now derive the momenta $m_{p q}$ :

$$
\begin{aligned}
m_{p q} & =\iint_{\text {region } 2} W\left(K_{x}, K_{y}\right) K_{x}{ }^{p} K_{y}{ }^{q} d K_{x} d K_{y} \\
& =\int_{0}^{2 \pi} \int_{x / r}^{2 k \sin \theta} W(\gamma, K) K^{p+q+1} \cos ^{p} \gamma \sin ^{q} \gamma d \gamma d K .
\end{aligned}
$$

We observe that $m_{02}=m_{20}$ and $m_{11}=0$. Then

$$
p\left(\tan \delta_{1}, \tan \psi_{1}\right)=\frac{1}{2 \pi m^{2}} \exp \left\{-\frac{\tan ^{2} \delta_{1}+\tan ^{2} \psi_{1}}{2 m^{2}}\right\} .
$$

Where $m^{2}=m_{02}{ }^{2}$. It should be mentioned that the same values of $m_{p q}$ also apply for a nondirectional spectrum or confused sea, and the probability distribution is then similar to the one used by Valenzuela et al. [19].

Using (3), (7), and (11) we can now determine $\sigma\left(\theta_{1}, \psi_{2}, \delta_{2}\right)$. In our calculations we assumed $\varepsilon=73-85 i$, which is valid for $L$-band [27]. For our purpose we are mainly interested in the sensitivity of $\sigma(\theta)$ to the changes in $\psi_{2}$ and $\delta_{2}$. Thus in Fig. 5 we plotted $\sigma(\theta)$ for the $\mathrm{HH}$ and VV polarizations as a function of $\theta$ for different values of $S$, $m$, and $\Phi$ where

$$
\begin{aligned}
S^{2} & =\tan ^{2} \psi_{2}+\tan ^{2} \delta_{2} \\
\tan \phi & =\tan \delta_{2} / \tan \psi_{2} .
\end{aligned}
$$

In Figs. 5(a)-5(d), the continuous line corresponds to the backscatter cross section in the absence of long waves. The dashed lines show the limits of the change in the backscatter cross section from one side to the other of a wave with a slope equal to 0.1 propagating perpendicularly to the line of flight (i.e., $\phi=90^{\circ}$ ). The dotted line corresponds to $\phi=45^{\circ}$. For $\phi=0^{\circ}$ the modulation is practically negligible. It can be clearly seen that there is an appreciable modulation of the $\mathrm{HH}$ backscatter at almost all look angles. To illustrate, for $\theta=20^{\circ}, m=0.05$, and $\phi=90^{\circ}$, the modulation is equal to $13 \mathrm{~dB}$, i.e., the slope toward the radar is $13 \mathrm{~dB}$ brighter than the slope away from the radar. For $\phi=45^{\circ}$ the modulation is equal to $7 \mathrm{~dB}$. The modula- 


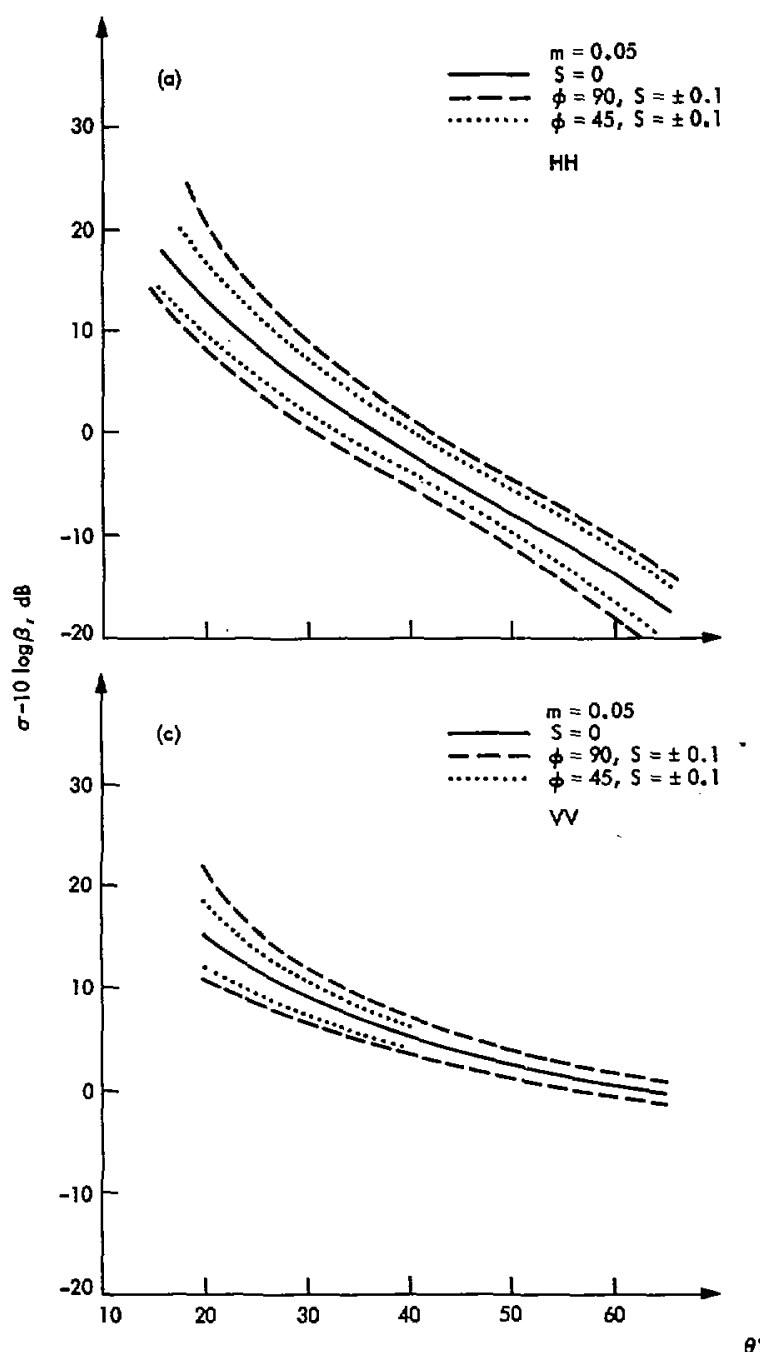

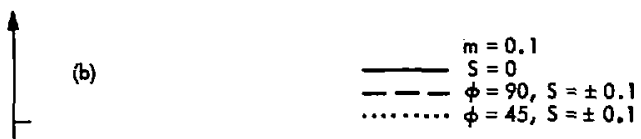

$\mathrm{HH}$
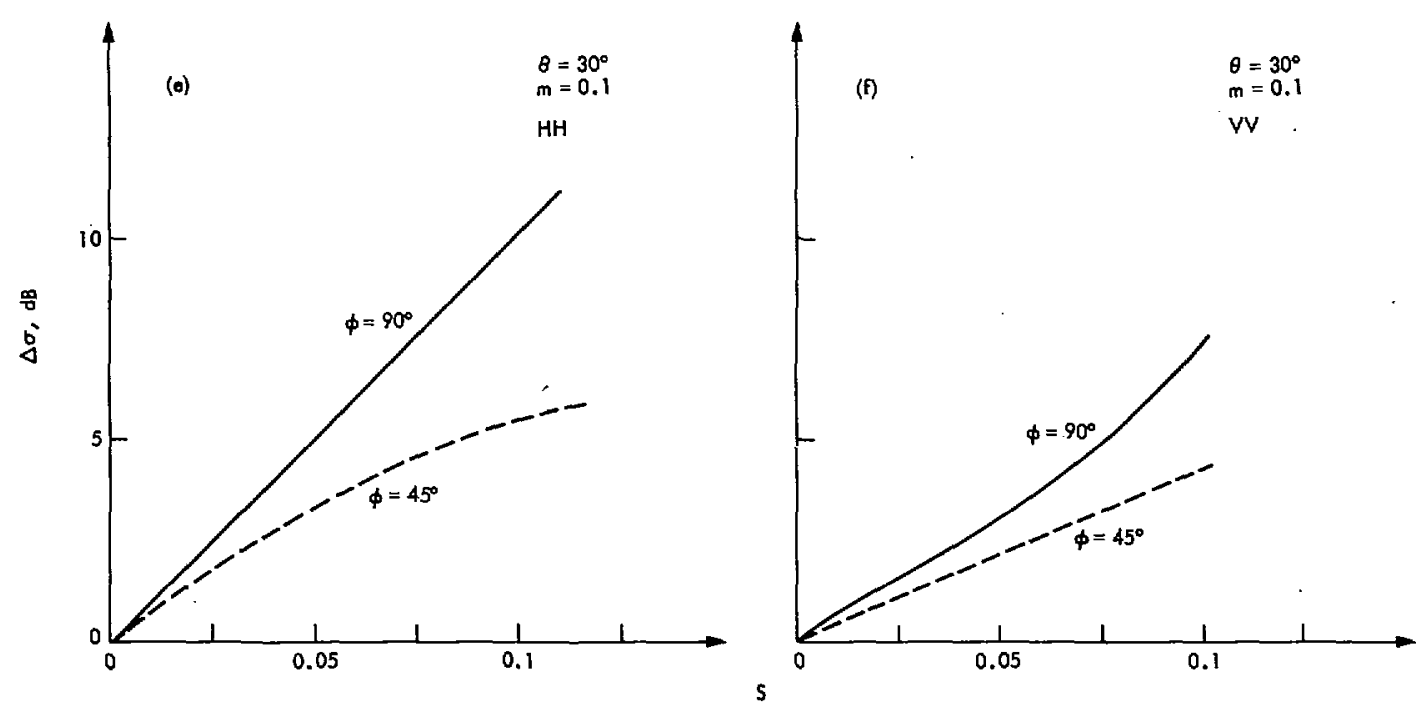

Fig. 5. (a) Plot showing dependence of backscattering cross section on look angle $\theta$ for $m=0.05$ and HH polarization Continuous line corresponds to case where $\phi=0^{\circ}$ or $S=0$. Dotted lines give upper and lower modulation limits Continuous line corresponds to case where $\phi=0^{\circ}$ or $S=45^{\circ}$. Dashed line corresponds to $\phi=90^{\circ}$. (b) Corresponds to $m=0.1$. (c) and (d) correspond to VV polarization. (e) and (f) show backscatter modulation $\Delta \sigma$ as function of $S$ and $\phi$ for fixed values of $\theta$ and $m$. 

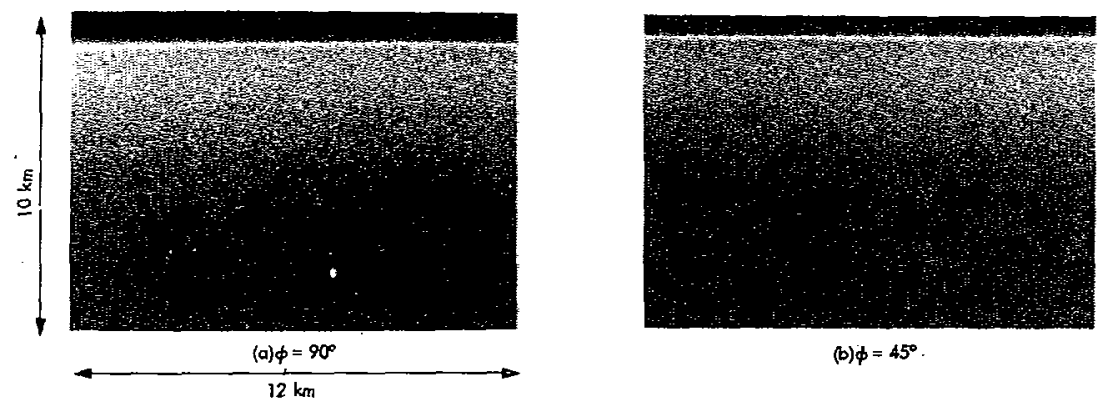

$(b) \phi=45^{\circ}$.

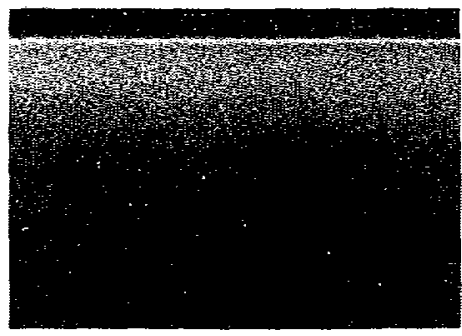

$(c) \phi=0^{\circ}$

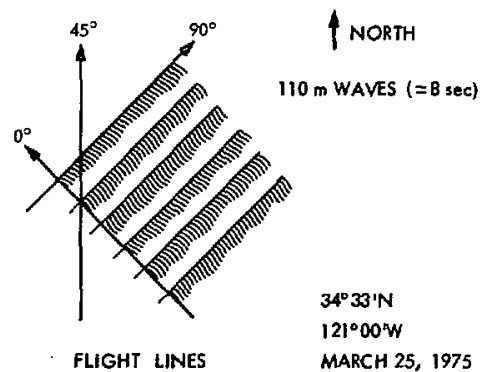

Fig. 6. Radar imagery of ocean waves taken from three different directions as shown in sketch in lower right side. Radar image corresponds to $10 \mathrm{~km}$ swath to right of flight line. $\Phi$ is angle between flight line and ocean wave vector. Ocean waves had wavelength of $110 \mathrm{~m}$ and significant wave height of $6 \mathrm{~m}$ as measured by surface buoy. Surface wind was 40 knots from northwest.
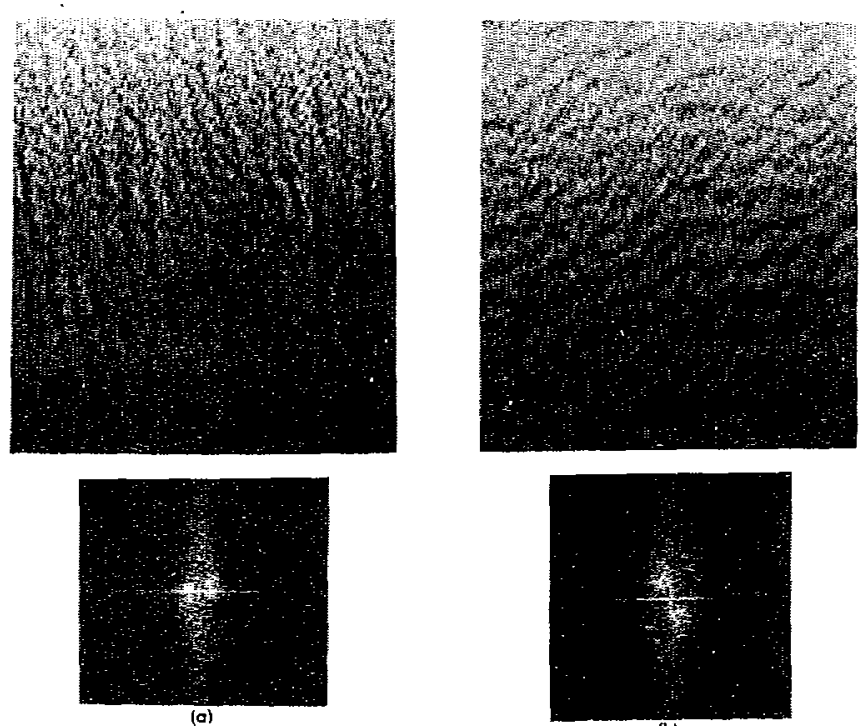

NORTH
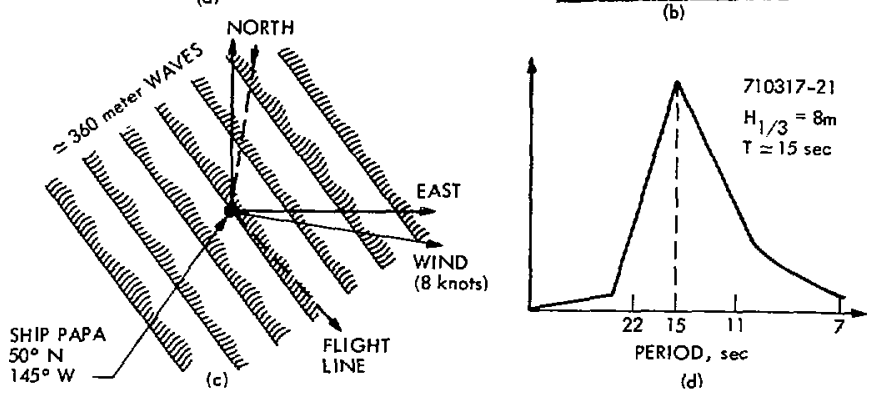

(d)

Fig. 7. Radar imagery of ocean swells taken from two different directions over station ship Papa in Gulf of Alaska. Swells had wavelength of $360 \mathrm{~m}$ and significant wave height of $8 \mathrm{~m}$ as measured by ship. Lower right corner graph is sketch of wave spectrum as measured by ship. In lower left corner is sketch of flight line. Also shown are 2-D Fourier transforms of image's intensity.

tion increases with $m$. In Figs. 5(e) and 5(f) we show the modulation $\Delta \sigma$ as a function of the slope $S$ for a fixed value of $m$.

A very important conclusion is that the $\mathrm{VV}$ backscatter is not as strongly modulated as the $\mathrm{HH}$ except at near vertical angles. It should be mentioned that Valenzuela et al. [19] did conclude in their study of radar scatterometry data that the RMS surface slope has less effect on the VV polarization backscatter. All imagery shown in this paper were taken at $\mathrm{HH}$ polarization.

From the above results we can conclude that the tilt modulation would provide enough change in the backscatter to allow the radar to image the surface. If this model is true then we would conclude that the radar will not image a wave propagating parallel to the line of flight. In Fig. 6 we show radar imagery taken of a fully developed sea where the local wind was about 40 knots, the significant wave height was about $6 \mathrm{~m}$ and the wave wavelength $110 \mathrm{~m}$. The three figures correspond to $\phi=0^{\circ}, 45^{\circ}$, and $90^{\circ}$ over exactly the same area. It is observed that at $\phi=0^{\circ}$ the waves were less visible than for $\phi=90^{\circ}$ which agrees with the tilt modulation model.

However, in Fig. 7, we show imagery taken with the same radar system over a different area where the local wind was about 8 knots, significant wave height was about $8 \mathrm{~m}$, and the swell wavelength $360 \mathrm{~m}$. The waves were clearly imaged for $\phi=0^{\circ}$ and $90^{\circ}$. Thus it seems that the tilt modulation model cannot explain the imagery in all cases. As a matter of fact, in most cases we obtained imagery of waves irrelevant of their direction relative to the flight line.

\section{Roughness Modulation Model}

Straining of small waves by the horizontal component of the orbital motion of larger waves is a well-known phenom- 
ena [20], [21]. This results in the ripples being shortened and steepened on the forward face of the large wave, and spread out and flattened on the backward face of the large wave. This leads to modulation of the surface roughness by the large wave. Other phenomena, such as wind-wave and wave-wave interaction, will also contribute to such a modulation. This modulation has been measured in a wave tank [22]-[24], however, there is no well developed theory that accounts for all the modulation factors in the case of open ocean waves. Thus in this section we will use an empirical approach.

A change in the surface roughness basically leads to a change of $\beta$ and $m$, which would result in a change in $\sigma$. It could also result in a change of the power dependence of the spectrum, i.e., $W(K) \sim 1 / K^{\nu \prime}$ with $v^{\prime} \neq 4$ but close to it. To illustrate let us take the case where $\beta=210^{-2}, m=0.1$, and $\theta=25$. The corresponding $\sigma_{\mathrm{HH}}$ is $-6 \mathrm{~dB}$. If we take $\boldsymbol{\beta}=0.510^{-2}$, then $m=0.05$ and $\boldsymbol{\sigma}_{\mathrm{HH}}$ would decrease by $8 \mathrm{~dB}$ to $-14 \mathrm{~dB}$. The change is similar for $\sigma_{\mathrm{vv}}$. Thus it seems that we could obtain appreciable modulation as a result of the change in roughness. However, we do not know how much $\beta$ will change in the real world.

It should be pointed out that if we assume that the small size waves that generate the scattered radar waves are not very directional, the modulation in the backscatter would have only a limited dependence on the direction of the wave relative to the line of flight. This model would then explain the imagery in Fig. 7. It should also be mentioned that in this model, both $\mathrm{VV}$ and $\mathrm{HH}$ polarization would provide enough backscatter modulation to generate an image.

In actuality, the modulation of the ocean surface backscatter cross section is most probably a composite of tilt and roughness modulation. These two factors could add up to increase, or subtract to decrease the modulation of the backscatter depending on the geometry. If the geometry is such that the slope of higher roughness is tilted toward the radar, then the composite modulation would be higher. The reverse would be true if the slope of higher roughness is tilted away from the radar.

The relative importance of the two types of modulation most probably depends on the local conditions. In the last five years we conducted more than a dozen flights in different locations and at different times. In most observations of the surface waves the image quality was not dependent on $\phi$. The one difference in the local condition over the area, where the data of Fig. 6 was taken relative to all other data we have, was the strong local wind. Thus we think that under such conditions, the surface roughness could have become homogeneous across the large wave thus eliminating the contribution of the roughness modulation.

\section{EFFect OF THE Waye Motion AND Temporal Changes}

It would seem that the somewhat random motion of the ocean surface would hinder the formation of a synthetic aperture. However, as we mentioned before, many coherent imaging radars did obtain imagery of the ocean waves and ocean currents. It is still not very clear what the effect is

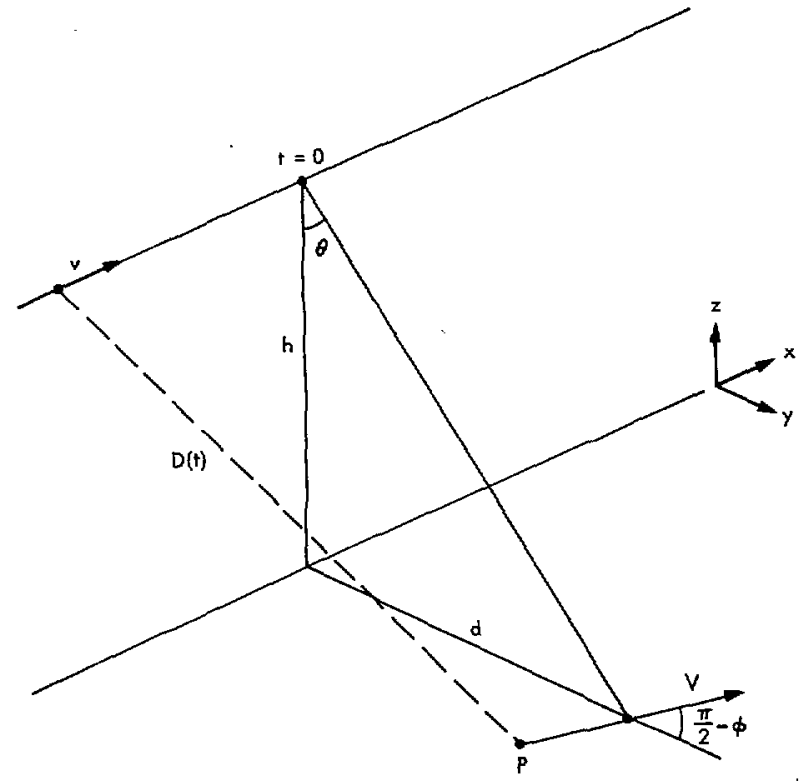

Fig. 8. Geometry which corresponds to case of moving target $P$.

of the surface motion and temporal change on the formation of the radar image. However, in this section, we will attempt to given an explanation of this effect. We will first derive the effect of the motion of a point target (i.e., one resolution element), then we will discuss the effect of the motion of a wave train. Finally we consider the temporal change of the surface structure in each resolution element and its effect on the imagery.

Let us consider the geometry shown in Fig. 8. $V$ is the velocity of the point target $P$ (i.e., one surface resolution element), and $v$ is the velocity of the platform. The time origin $t=0$ corresponds to the time when $P$ is viewed with a $90^{\circ}$ angle. By simple algebraic calculation we can derive that the distance $D(t)$ is given by

$$
D^{2}(t)=h^{2}+(1-a) d^{2}+(1+b) v^{2}(t+\tau)^{2}
$$

where

$$
\begin{aligned}
& a=\frac{V^{2} \sin ^{2} \phi}{V^{2}+v^{2}-2 v V \cos \phi} \simeq\left(\frac{V \sin \phi}{v}\right)^{2} \\
& b=\frac{V^{2}-2 v V \cos \phi}{v^{2}} \simeq-2 V \cos \phi / v \\
& \tau=\frac{V d \sin \phi}{V^{2}+v^{2}-2 v V \cos \phi} \simeq \frac{V d}{v^{2}} \sin \phi .
\end{aligned}
$$

We assume $V \ll v$. In the case of a stationary target $(V=0)$, then $a=b=\tau=0$. Thus the first order effects of the motion are

1) to displace the image in the $y$ direction by the amount

$$
\Delta y=\Delta d=-\frac{a}{2} d=-\frac{1}{2}\left(\frac{V \sin \phi}{v}\right)^{2} d
$$

2) to displace the image in the $x$ direction by the amount

$$
\Delta x=-v \tau=-\frac{V}{v} d \sin \phi
$$



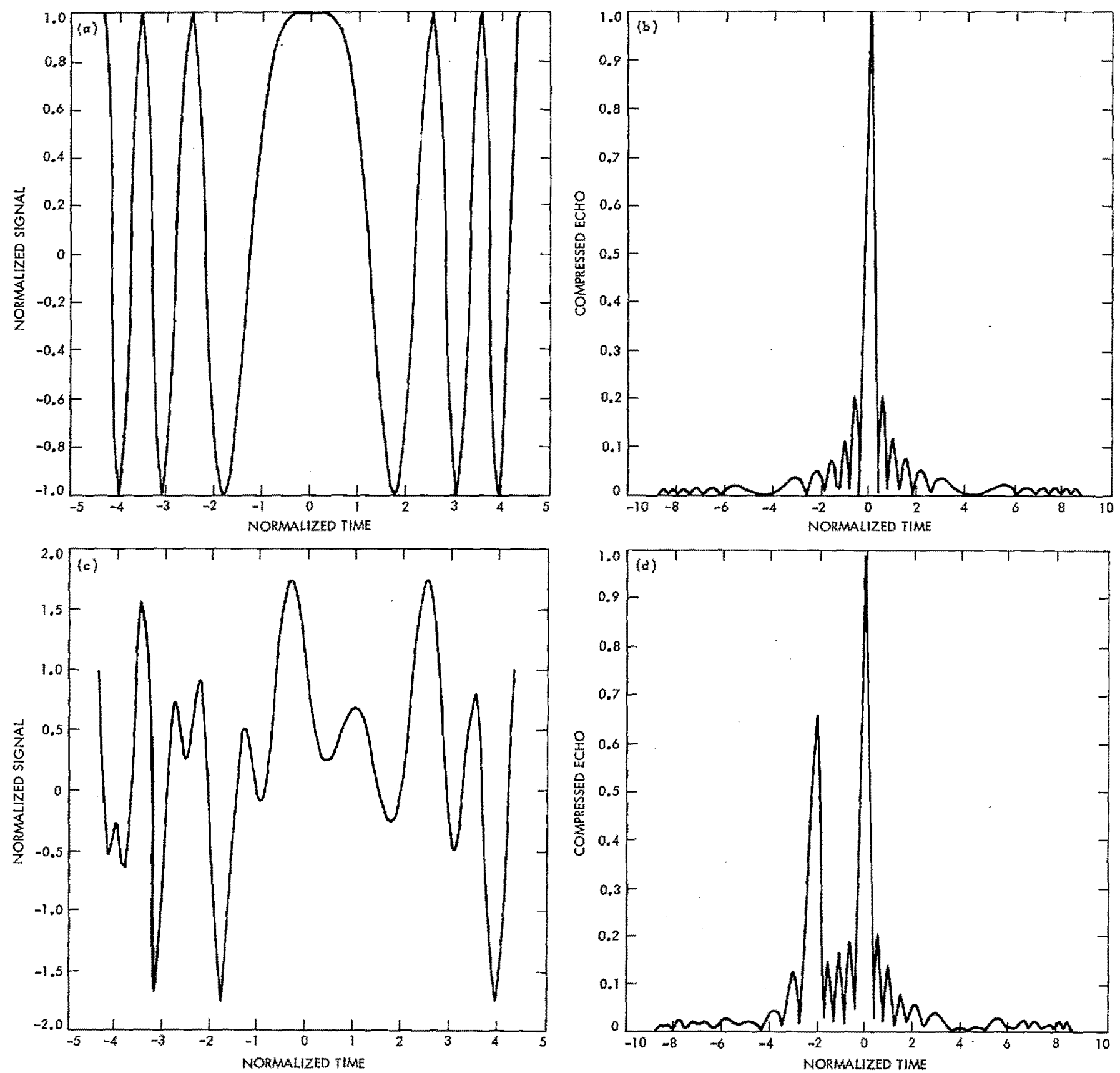

Fig. 9. Normalized signal history and compressed return. (a) and (b) Case of one resolution element. (c) and (d) Case of two neighboring resolution elements of different strength (1 and 0.75$)$. Signal history curve is locus of real value of signal from successive radar pulses.

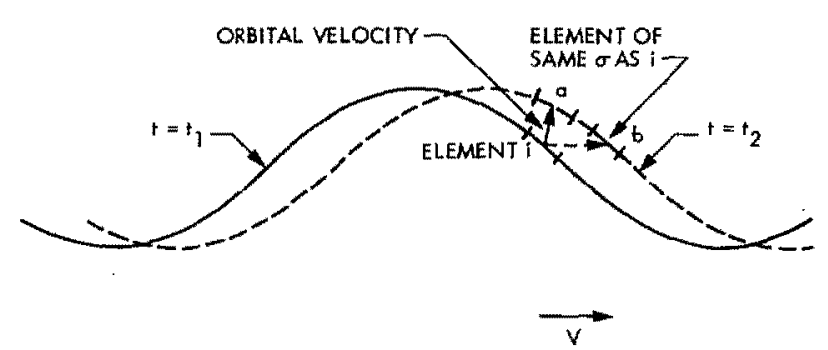

Fig. 10. Wave position at two successive times $t_{1}$ and $t_{2}$. Resolution element $i$ of constant backscatter cross section moves in same direction as wave (to position $b$ ) even though particles themselves follow orbital velocity (to position a). From radar point of view, surface element is characterized by backscatter cross section and not by particles which form it.
3) to change the apparent velocity of the platform by

$$
\frac{\Delta v}{v}=\frac{b}{2}=-\frac{V}{v} \cos \phi .
$$

This has a direct effect on the focusing during the processing of the synthetic aperture data. To illustrate, let us take $V=12.5 \mathrm{~m} / \mathrm{s}, v=250 \mathrm{~m} / \mathrm{s}$, and $d=5000 \mathrm{~m}$, which correspond to typical experimental values; then:

$$
\begin{aligned}
|\Delta y| & \simeq 6 \sin ^{2} \phi \quad \text { in meters } \\
\Delta x & =250 \sin \phi \quad \text { in meters } \\
\left|\frac{\Delta v}{v}\right| & \simeq \cos \phi / 20 .
\end{aligned}
$$


Thus the only appreciable error is the displacement $\Delta x$ along the line of flight which is maximum when $\phi=90^{\circ}$.

The second step is to consider the effect of the wave motion. To evaluate this effect let us describe how the radar image is generated in azimuth. This is particularly important because the radar uses the Doppler information to generate the synthetic aperture. If a point target $P$ is assumed fixed, then the corresponding history of the real part of the signal (i.e., $\sim \cos \Phi(t)=\cos (4 \pi D(t) / \lambda)$ is shown in Fig. 9(a). The history of the real part of the signal is in actuality a succession of points because the radar is pulsed and each point corresponds to one pulse. Thus, the signal history is a pulse to pulse change of the real part of the signal. This signal history is recorded and then compressed to generate one peak (i.e., one point or pixel).

Now let us assume two neighboring resolution elements of different backscatter cross section. The composite signal history will consist of the history of the real part of the vectorial sum of two echoes (Fig. 9(b)) which have different amplitudes and are displaced in time relative to each other. After compression we get two neighboring peaks of amplitudes proportional to the backscatter of the corresponding resolution element. It is important to mention that usually the synthetic aperture is formed over a period of the order of one second.

Now let us consider an ocean wave at time $t=t_{1}$. The wave surface can be divided in resolution elements $i$ each one of them characterized by a backscatter cross section $\sigma_{i}\left(t_{1}\right)$. A short time later, at $t=t_{2}$ (Fig. 10) the wave has a new position. The instantaneous motion of the water particles is the well known orbital motion. However, the area of constant backscatter cross section (i.e., constant slope and statistically constant roughness) is now in the position shown in Fig. 10, i.e., it moved parallel to the wave motion. The fact that the water particles in the resolution element $i$ at time $t_{2}$ are not the same as at time $t_{1}$ is irrelevant as long as the backscatter cross section is still the same. Thus during the time the image is formed, which is of the order of $1 \mathrm{~s}$, the surface appears to have moved with the phase velocity of the wave. This is true for both the tilt modulation and roughness modulation. So the net result is a displacement of the whole image along the line of flight (from (15)). It is important to mention that this displacement is maximum when $\phi=0$, i.e., the waves move perpendicularly to the line of flight. However, such a displacement along the crest line is completely indistinguishable, except in the case where fixed boundaries are present. Thus the maximum visible distortion (i.e., displacement) occurs for waves at about $45^{\circ}$ from the flight line.

Another phenomenon which also leads to image displacement is the parametric interaction of the radar wave with the ocean wave which scatters it. This is discussed in detail in the next section.

Finally, let us consider the effect of temporal changes other than the average motion in one resolution element. Each resolution element can be considered a linear superposition of an infinite number of waves of a wavelength up

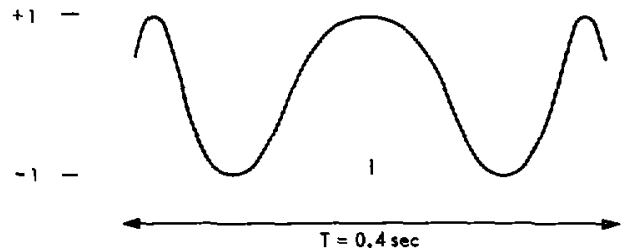

(a)

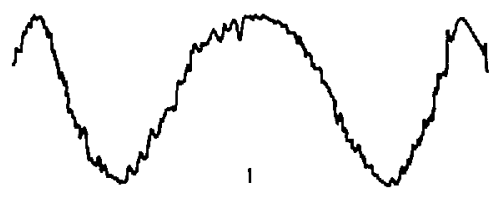

(b)

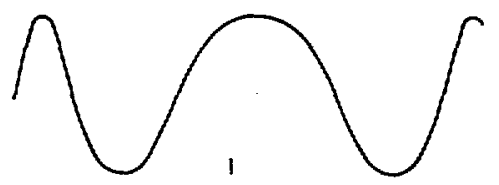

(c)

Fig. 11. (a) Typical signal history for aircraft imaging radar. (b) Effect of high frequency small magnitude perturbation. (c) Effect of low frequency large perturbation.

to twice the resolution $r$. The main question is how does the change in the surface affect the phase of the resultant back scattered wave. An exact solution of this problem is very complicated, thus we will use a simplified model combined with a numerical example to illustrate the magnitude of this effect.

Let us consider a $\lambda=25 \mathrm{~cm}$ imaging radar mounted on an aircraft flying at a velocity $v=250 \mathrm{~m} / \mathrm{s}$ and at an altitude $h=10000 \mathrm{~m}$. These numbers are typical for the imagery shown in this paper. To achieve a resolution $r=25 \mathrm{~m}$, the length $L$ of the synthetic aperture must be $L=\lambda h / r \cdot 100 \mathrm{~m}$ which requires a time $T=L / v=0.4 \mathrm{~s}$ to be generated. The corresponding real signal history is shown in Fig. 11(a). The main question is how much phase change is induced as a result of the temporal change in the surface topography. Waves of periods less than $0.4 \mathrm{~s}$ have a wavelength less than $25 \mathrm{~cm}$ and their wave height is probably less than a few centimeters, which is small relative to the radar wavelength. Thus their effect is to add a high frequency small fluctuation to the signal history (Fig. 11(b)). These perturbations will average out during compression resulting, at worst, in a slight degradation in the resolution. Waves of periods larger than a few seconds might have a height equal to or larger than the radar wavelength; however, they add only a low frequency perturbation (Fig. 11(c)) which results in a displacement of the image as mentioned previously. Thus it seems that the temporal changes do not destroy the coherency of the synthetic aperture as long as extremely high resolutions are not required. A numerical analysis of 
the effect of random phase on the generation of the synthetic aperture also gave the same conclusion [28].

\section{Parametric Interaction Effects}

As mentioned previously the radar waves scatter mainly from the ocean waves which satisfy the Bragg condition, i.e., wave vector matching. The resulting condition is the well-known Bragg condition

$$
\lambda=2 \Lambda \sin \theta
$$

where $\lambda$ is the radar wavelength and $\Lambda$ the ocean wave wavelength. Due to the fact that the ocean wave is moving and has an angular frequency $\Omega$, a second condition, the frequency matching condition, is required, i.e., the backscattered wave frequency is equal to the incident wave frequency minus the ocean wave frequency:

$$
\omega_{s}=\omega_{i}-\Omega
$$

where $\omega_{s}$ and $\omega_{i}$ are, respectively, the angular frequencies of the scattered and incident radar wave. The above two conditions are the well-known parametric interactions conditions. In scatterometry experiments, the second condition is of little importance because $\Omega \ll \omega_{i}$. However in over-thehorizon radars, the frequency matching condition is critical [11] because the echo is mixed with a stable local oscillator to measure the frequency shift induced by the motion of the ocean wave. In the case of imaging radar, the echo is also mixed with the local oscillator. Thus a term $-\Omega t$ has to be added to (2). This would lead to an image displacement $\Delta x^{\prime}$ equal to

$$
\Delta x^{\prime}=-\frac{h}{v} \frac{\lambda}{2 T}
$$

where $T$ is the wave period. If the radar scattering is mainly from short gravity waves, then

and

$$
\Lambda=g T^{2} / 2 \pi
$$

$$
\Delta x^{\prime}=-\left(\frac{h}{v}\right)(\lambda g \sin \theta / 4 \pi)^{1 / 2} .
$$

To illustrate, if $\lambda=0.25 \mathrm{~m}, \theta=30^{\circ}$, and $h / v=40 \mathrm{~s}$, then $\Lambda=0.25 \mathrm{~m}$ and $\Delta x^{\prime} \simeq 13 \mathrm{~m}$. It should be emphasized that this displacement is along the flight line and is independent of the relative direction of the waves.

The displacement $\Delta x^{\prime}$ decreases with $\lambda$, however below a certain value, the scattering will result mainly from capillary waves and (18) is not valid any more. As a matter of fact $\Lambda / T^{2}$ will increase drastically leading to a fast increase of $\Delta x^{\prime}$ for imaging radars operating in bands higher than the $X$-band.

It should be mentioned that the parametric effect has been considered as a possible reason for the formation of radar ocean waves imagery. Due to the change in the local tilt, the frequency shift $\Omega$ is different for the echoes coming from a different part of a wave. This would result in a modulated mismatching with the local oscillator, which would result in a modulated image. However, calculations by Evans and Elachi [29], showed that this effect is negligible.

\section{EFFECT OF THE ORBITAL VeLOCITY}

Another model that was suggested to explain the radar imagery of ocean waves is based on the effect of the orbital velocity on the formation of the radar image [6], [30]. It basically states that the scattered signal from a resolution element $i$ is Doppler shifted by the orbital velocity $C$ at that point. To illustrate, let us assume a large angle of incidence. In this case, only the horizontal component of the orbital velocity has to be accounted for. Thus point $\dot{P}$ will be displaced along the line of flight by a value (see (15))

where

$$
\Delta x=d \sin \phi C(x) / v
$$

$$
C(x)=C_{0} \sin (K x \cos \phi)
$$

with adequate choice of the origin. So the position of point $P$ in the image is

$$
x^{\prime}=x+\Delta x=x+\frac{d C_{0}}{v} \sin \phi \sin (K x \cos \phi) .
$$

If we assume the surface has a linear density of scatterers $\rho(x)$ (i.e., backscatter cross section is $\sim \rho(x) d x$ ), then the brightness density in the image plane $\rho\left(x^{\prime}\right)$ is related to $\rho(x)$ by

which implies

$$
\rho\left(x^{\prime}\right)\left|d x^{\prime}\right|=\rho(x)|d x|
$$

$$
\begin{aligned}
\rho\left(x^{\prime}\right) & =\rho(x)\left|\frac{d x}{d x^{\prime}}\right| \\
& =\frac{\rho(x)}{\left|1+\frac{K d C_{0}}{v} \cos \phi \sin \phi \cos (K x \cos \phi)\right|}
\end{aligned}
$$

and the average brightness over a resolution element in the radar image at point $X$ is proportional to

$$
I(X)=\int_{X}^{X+r} \rho\left(x^{\prime}\right) d x=\int_{X}^{X+r} \frac{\rho(x) d x}{\left|1+M \cos \left(K^{\prime} x\right)\right|}
$$

where

$$
\begin{aligned}
M & =\frac{K d C_{0}}{2 v} \sin 2 \phi=\frac{\pi d}{v T} S \sin 2 \phi \\
K^{\prime} & =K \cos \phi \\
S & =\text { slope of the wave } \\
T & =\text { period of the wave. }
\end{aligned}
$$

The factor $M$ can be considered as a modulation factor. Thus even if $\rho(x)=\rho=$ constant, the image intensity is modulated by the effect of bunching of the scatterers as a result of the velocity modulation. The image modulation vanishes for $\phi=0^{\circ}$ and $90^{\circ}$ and is maximum for $\phi=45^{\circ}$.

To illustrate the effect of the value of $M$ for sufficiently small $r$, let us consider the function

$$
f(\xi)=\frac{1}{|1+M \cos \xi|} .
$$




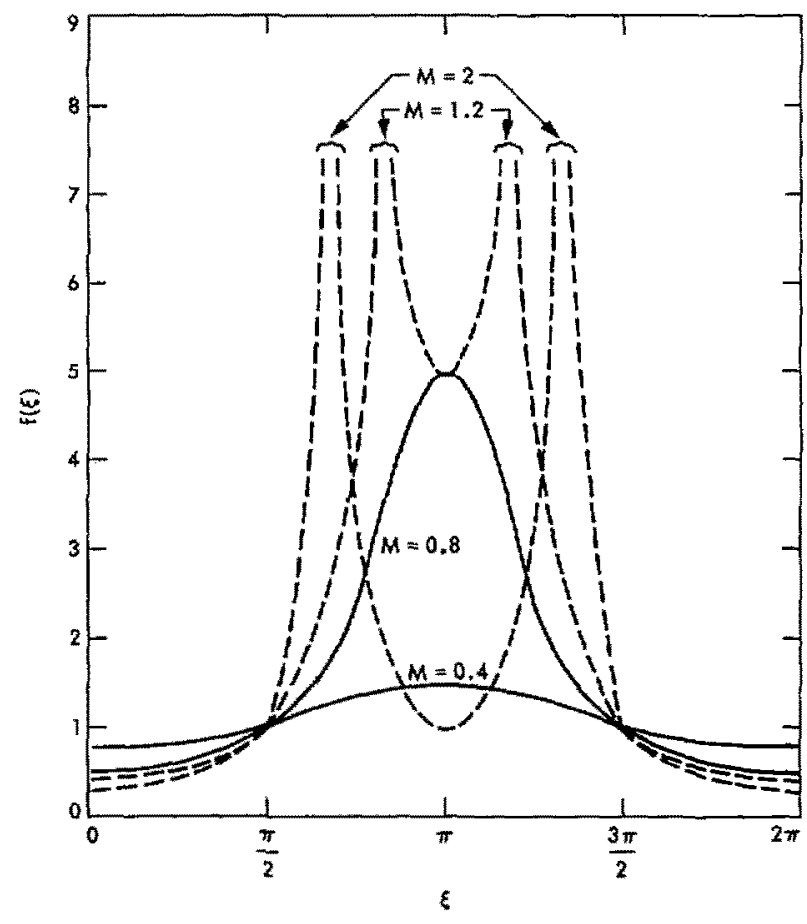

Fig. 12. Plot of $f(\xi)$ for different values of $M$.

In Fig. 12 we plotted the value of $f(\xi)$ for different values of $M$. This shows that for all values of $M$, the image is modulated with a period equal to $\Lambda / \cos \phi$; however the modulation is not sinusoidal. It can be easily seen that the quantitative interpretation of the image becomes complicated. It also implies that waves where $\phi=0^{\circ}$ and $90^{\circ}$ cannot be imaged at large incidence angles. However, most probably all the previously discussed effects are present simultaneously, and their relative importance is dependent on the local conditions.

\section{CONCLUSION}

As we mentioned in the introduction, the field of imaging radar oceanography is a new field of study and more experimental and theoretical work is still needed to achieve a full understanding of the radar image. In this paper, we discussed a number of models that seem most promising and popular, and presented some numerical and experimental data for or against their support. A fortunate result is that each one of the models discussed in this paper gives a modulation which has a different dependence on the angle $\phi$ between the line of flight and the wave direction as sketched in Fig. 13. Thus a series of experimental flights over different sea states where, during each flight, imagery is taken at different values of $\phi$, would allow us to determine the importance or the validity of the different models.

\section{ACKNOWLEDGMENT}

The authors would like to thank Dr. S. Lazanoff for providing some of the ground truth data, the NASA EODAP office for its support of this work, and the members

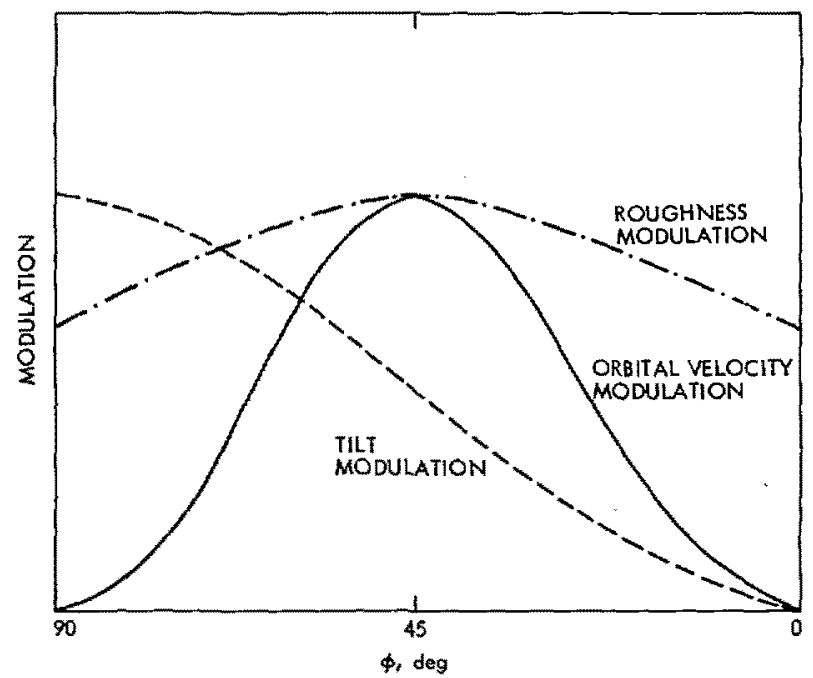

Fig. 13. Sketch of backscatter modulation dependence on angle between flight line and wave direction for three different models considered in this paper.

of the Seasat SAR team and Seasat project office for many enlightening discussions. The authors also acknowledge the help of Mr. D. Evans (JPL) in providing Fig. 9.

\section{REFERENCES}

[1] W. E. Brown, Jr., C. Elachi, and T. W. Thompson, "Oceanographic observation with imaging radar," URSI Fall Meeting, Boulder, CO, 1973.

[2] -, "Radar imaging of ocean surface patterns," URSI Fall Meeting, Boulder, CO, 1974.

[3] - "Radar imaging of ocean surface patterns," J. Geophys. Res., vol. 81 , pp. $2657-2667,1976$.

[4] C. Elachi" "Wave patterns across the north Atlantic on September 28,1974 , from airborne radar imagery," J. Geophys. Res., vol. 81 , pp. $2655-2656,1976$

[5] L. I. Moskowitz, "The feasibility of ocean current mapping via synthetic aperture radar methods," Proc. Fall Conv. Amer. Soc, Photogrammetry, pp. $760-771$, Oct. 1973.

[6] T. R. Larson and J. W. Wright, "Imaging ocean current gradients with synthetic aperture radar," URSI Fall Meeting, Boulder, $\mathrm{CO}$, 1974 .

[7] J. W. Goodman, Introduction to Fourier Optics. San Francisco: McGraw-Hill, 1968.

[8] A.W. Rihaczek, Principles of High Resolution Radar. New York: MoGraw-Hill, 1969.

[9] R. O. Harger, Synthetic Aperture Radar Systems. New York: Academic, 1970.

[10] D. E. Barrick, "Rough surface scattering based on the specular point theory," IEEE Trans. Antennas Propagat, vol. AP-16, pp. $449-454,1968$.

[11] - "First order theory and analysis of MF/HF/VHF scatter from the sea," IEEE Trans. Antemas Propagat., vol. AP-20, pp. 2-10, 1972 .

[12] K. Hasselman, "Determination of ocean wave spectra from Doppler radio return from the sea," Nature Phys. Sci., vol. 229, pp. 16-17, 1971 .

[13] K. Krishen, "Correlation of radar backscatter cross-sections with ocean wave height and wind velocity," J. Geophys. Res., vol. 76, pp. $6528-6539,1971$.

[14] J. C. Daley, "Wind dependence of radar sea return," $J$. Geophys. Res., vol. 78, pp. $7823-7833,1973$.

[15] G. R. Valenzuela, "The effect of capillary and resonant interaction on the second order Doppler spectrum of radar sea echo," $J$. Geophys. Res., vol. 79, pp. 5031-5037, 1974.

[16] J. W. Wright, "Backscattering from capillary waves with application to sea clutter," IEEE Trans. Antentas Propagat., vol. AP-14, pp. 749-754, 1966 .

[17] G. R. Valenzuela, "Scattering of electromagnetic waves from a tilted slightly rough surface," Radio Sci., vol. 3, pp. 1057-1066, 1968. 
[18] K. Hasselman and M. Schieler, "Radar backscatter from the sea surface," paper presented at the 8th Symposium of Naval Hydrodynamics, U.S. Navy, Pasadena, CA, 1970.

[19] G. R. Valenzuela, M. B. Laing, and J. C. Daley, "Ocean spectra for the high frequency waves as determined from airborne radar measurements," J. of Marine Res., vol, 29, pp. 69-84, 1971.

[20] B. Kinsman, Wind Waves. Englewood Cliffs, N.J.: PrenticeHall, 1965.

[21] O. M. Phillips, The Dynamics of the Upper Ocean. Cambridge: Uníversity Press, 1966.

[22] O. H. Shemdin, R. J. Lai, A. Reece, and G. Tober, "Laboratory investigations of white caps, spray and capillary waves," Technical Rep. No. 11, COEL, Univ. of Florida, 1972.

[23] W. C. Keller and J. W. Wright, "Microwave scattering and the straining of wind generated waves," Radio Science, vol. 10, pp.
$139-147,1975$.

[24] J. Wu, "Correlation of micro- and macroscopic structures of wind waves," IEEE Ocean 75 Conference, La Jolla, 1975.

[25] C. M. Cox and W. H. Munk, "Measurement of the roughness of the sea surface from photographs of the sun's glitter," $J$. Opt. Soc. of $A m_{.,}$vol. 44, pp. 838-850, 1954.

[26] M. S. Longuet-Higgins, "Statistical analysis of a random moving surface," Phil. Trans. Roy. Soc., vol. 249, pp. 321-387, 1957.

[27] J. A. Saxton and I. A. Lane, "Electrical properties of sea water," Wireless Eng., vol. 29, pp. 269-275, 1952.

[28] C. Elachi and D. Evans, "Effects of random phase changes on the formation of synthetic aperture radar imagery, ${ }^{12}$ this issue, $p p$. 149-153.

[29] D. Evans and C. Elachi, unpublished.

[30] J.W. Sright, private communication (memo, dated 1973).

\title{
Three-Dimensional Vector Modeling and Restoration of Flat Finite Wave Tank Radiometric Measurements
}

\author{
WILLIAM M. TRUMAN, STUDENT MEMBER, IEEE, CONSTANTINE A. BALANIS, SENIOR MEMBER, IEEE, \\ AND JOHN J. HOLMES, STUDENT MEMBER, IEEE
}

\begin{abstract}
In this paper, a three-dinensional Fourier transform inversion method describing the interaction between water surface emitted radiation from a flat finite wave tank and antenna radiation characteristics is reported. The transform technique represents the scanning of the antenna mathematically as a correlation. Computation time is reduced by using the efficient and economical fast Fourier transform algorithm. To verify the inversion method, computations have been made and compared with known data and other available results. The technique has been used to restore data of the finite wave tank system and other available antenna temperature measurements made at the Cape Cod Canal. The restored brightness temperatures serve as better representations of the emitted radiation than the measured antenna temperatures.
\end{abstract}

\section{INTRODUCTION}

$T^{\circ}$ TO MAKE precise measurements of the radiometric brightness temperature of a target (and thereby infer certain physical parameters), one must be able to mathematically model the interaction between the electromagnetic radiation properties of the antenna and the incident radiation from the environment. This interaction can be described by Fredholm integral equations of the first kind which are extremely unstable. This instability has

Manuscript received August 22, 1975; revised January 15, 1976. This research was supported by the National Aeronautics and Space Administration Langley Research Center, Hampton, VA, under Grant NGR 49-001-056.

The authors are with the Department of Electrical Engineering, West Virginia University, Morgantown, WV 26506. been studied in considerable detail by investigators in many fields. Twomey [1] and Phillips [2] have devised matrix filtering techniques to stabilize the solution. Although these matrix methods are not without merit, Bracewell and Roberts [3] have demonstrated the value of a successive substitution solution. Assuming that the intensity of the emitted radiation of the environment can be represented in scalar form, they have shown that the antenna is only capable of responding to frequency components below a cutoff determined by the antenna aperture. The high frequency components of the emission function are invisible to the antenna. The low frequency components are accepted but their relative magnitude is altered according to the system (antenna) filtering characteristics. Inversion through the method of successive restorations leads to the principal solution [3], in which frequency components accepted by the antenna have been restored to their original values, but the rejected components are not represented in the solution. The work done by Bracewell and Roberts was, however, more applicable to astronomical observations than to general microwave radiometric measurements. They assumed that the antenna was very efficient and that the sidelobes and backlobes could be neglected, which is not always the case. They also used a scalar representation of the interaction between the antenna radiation characteristics and the emission by the target. 